\title{
On the Numerical Modeling of FENE-CR Viscoelastic Droplet Impact Dynamics by the Volume Of Fluid Method
}

\author{
Moussa Tembely ${ }^{1 \pm}$, Damien Vadillo ${ }^{2}$, Ali Dolatabadi ${ }^{1}$, Arthur Soucemarianadin ${ }^{3}$ \\ ${ }^{ \pm}$corresponding author: moussa.tembely@concordia.ca \\ ${ }^{1}$ Department of Mechanical Engineering, Concordia University, Montreal, Canada \\ 2 AkzoNobel, Research, Development and Innovation, Gateshead, UK \\ 3 Université de Grenoble-Alpes, CNRS, LEGI, F-38000 Grenoble, France
}

\begin{abstract}
In this paper, a numerical modeling of the impact, spreading, and eventually rebound of a viscoelastic droplet is reported. The numerical model is based on the volume of fluid (VOF) method coupled with the FENE-CR constitutive equations, and accounts for both the surface tension and the substrate wettability. The FENE-CR constitutive equations are used to model the polymer solution, while taking advantage of its rheological characterization. The comparison is performed between droplets of Newtonian solvent and a monodisperse polymer solution. The droplet impact on both hydrophilic and superhydrophobic substrate is analyzed through a detailed analysis of the spreading diameter evolution. It is found that while the droplet kinematic phase seems independent of the substrate and fluids properties, the recoiling phase is highly related to all of them. In addition, the model infers a critical polymer concentration above which the droplet rebound from a superhydrophobic substrate is suppressed. The simulation is of particular interest to ink-jet processing, and demonstrates the capability of the model to handle complex non-Newtonian droplet dynamics.
\end{abstract}

Keywords: Droplet Impact, Viscoelastic, Two-phase Flow, Volume of Fluid method (VOF)

*Initially presented at the 4th Micro and Nano Flows Conference, London, UK, 7-10 September 2014. 


\section{Introduction}

Owing to their versatility, non-Newtonian fluids play a key role in a wide range of applications. Among these applications are inkjet printing, lab-on-chip, digital display (pLED), biological assays (Clemens et al. 2004, Xu and Attinger, 2008). The liquids involved in those processes are likely to exhibit complex micro-structure and non-Newtonian properties, such as viscoelasticity. The flexible polymer composing those liquids may alter considerably the liquid flow behavior compared to the Newtonian one, as highlighted by Amarouchene et al. (2001).

Fundamental understanding of the droplet dynamics of complex fluids is a key element for future breakthrough in the growing domain of micro-fabrication and micro-fluidics (Singh et al., 2010). Regarding droplet impact, the question of whether the droplet will be deposited on the substrate or eventually rebound is of particular interest, and a full understanding of the entire droplet impact dynamics is required (Mohammadi et al., 2017). Although Newtonian droplet impact has been extensively studied in the literature, the non-Newtonian viscoelastic droplet impact, despite of the plethora of applications, is loosely documented both experimentally and numerically, even though recent works, showing an increasing interest for these fluids, make them an active research area.

Over the past decades, different techniques have been developed to address non-Newtonian free surface flow modeling. A finite element method has been pioneered by Crochet and Keunings, (1982) to model the die swell of an Oldroyd-B fluid through an ad-hoc iterative technique, for the free surface, based on the kinematic condition. The split Lagrangian-Eulerian method has been extended by Morrison and Harlen (2010) to study viscoelastic jet breakup. Recently Tomé et al. $(2002,2010)$ simulated free surface flow using both maker and cell (MAC) and finite difference (FD) techniques. In addition, a mesh free method using smoothed particle hydrodynamics (SPH) technique has been applied to model an Oldroyd-B drop impact in 2D, while neglecting the surface tension (Fang et al., 
2006). These different techniques neglect both the surface tension and the dynamic contact angle during spreading along with the presence of air. In addition, it is worth noting the interface capturing techniques to model non-Newtonian free surface flow such as the level set method (Yu et al., 2007) or the phase field method (Yue and Feng, 2012) where the viscoelastic fluid is described by the Oldroyd-B model. Finally it is worth mentioning a one-dimensional approach (Tembely al., 2012) to model free surface flow of non-Newtonian fluid using an Arbitrary Lagrangian-Eulerian (ALE) Methods, which shows good results in modeling filament thinning using FENE-CR model.

Although the low viscosity dilute polymer are of particular interest in inkjet fluids [Dong et al. 2006, Hoath et al. 2014], it is only recently that they have been experimentally investigated using a controlled stretching rheometer (Vadillo et al. 2010), and the present work will benefit from the rheological characterization of these fluids to optimize the simulation.

Most of the numerical studies consider viscoelastic internal flow, but few consider droplet interaction with a solid substrate. Within the Volume of Fluid (VOF) framework, the present model will account for both the surface tension and the substrate wettability, where a dynamics contact angle is implemented, as opposed to a static contact angle model. The impacted droplet viscoelasticity effect is modeled using a single mode FENE-CR model in axisymmetric configuration.

The outline of the paper is as follows. Section 2 presents the governing equations while the rheological characterization is presented in Section 3. Section 4 details the numerical results and discussions along with the main features of Newtonian and viscoelastic droplet impact on hydrophilic and superhydrophobic substrates. Finally, the conclusions are drawn in Section 5.

\section{Governing equations}

The fluid motion and droplet impact are modeled using Navier-Stokes equations, assuming the 
liquid of density $(\rho)$, solvent viscosity $\left(\eta_{\mathrm{s}}\right)$ and surface tension $(\gamma)$, to be incompressible, and the flow to be laminar. The continuity and momentum equations accounting for the viscoelastic term as an extra stress tensor $\left(\boldsymbol{\sigma}_{p}\right)$ to be numerically solved in the VOF formulation are:

$$
\begin{gathered}
\nabla \cdot \mathbf{V}=0 \\
\frac{\partial(\rho \mathbf{V})}{\partial t}+\nabla \cdot(\rho \mathbf{V V}) \\
=-\nabla p+\rho \mathbf{g}+\nabla \cdot\left(\eta_{S} \nabla \mathbf{V}\right)+\nabla \cdot \boldsymbol{\sigma}_{p}+\gamma \kappa \nabla \alpha
\end{gathered}
$$

where $\mathbf{V}, p, \mathbf{g}, \kappa$ are the velocity, pressure, gravity acceleration, and liquid-gas curvature, respectively.

The polymer contribution of the viscoelastic fluid models is described by a Finitely Extensible Nonlinear Elastic (FENE) dumbbell model which makes use of the conformation tensor A, and the stress tensor reads (Chilcott and Rallison, 1988):

$$
\boldsymbol{\sigma}_{p}=G f(R)(\mathbf{A}-\mathbf{I})
$$

where $G$ is the elastic modulus, $f(R)$ is the finite extensibility factor related to the finite extensibility parameter $L$, representing the ratio of a fully extended polymer (dumbbell) to its equilibrium length and $R=\operatorname{Tr}(\mathbf{A})$,

$$
f(R)=\frac{1}{1-R / L^{2}}
$$

The conformation tensor evolution expresses as follows:

$$
\frac{d \mathbf{A}}{d t}-\nabla \mathbf{V}^{T} \cdot \mathbf{A}-\mathbf{A} \cdot \nabla \mathbf{v}=-\frac{f(R)}{\lambda}(\mathbf{A}-\mathbf{I})
$$

where $\lambda$ is the relaxation time while $\mathbf{I}$ is the identity tensor. An additional parameter $c=G \lambda / \eta_{\mathrm{s}}$ is often 
introduced, which may be interpreted as a measure of the concentration (volume fraction) of dumbbell molecules.

The two phases liquid and the gas are defined by the volume phase fraction $(\alpha)$ which is advected by the flow, and the transport equation using the interface compression method reads as follows:

$$
\frac{\partial \alpha}{\partial t}+\mathbf{V} \nabla \alpha+\nabla \cdot\left[\mathbf{V}_{c} \alpha(1-\alpha)\right]=0
$$

The compression velocity $\boldsymbol{V}_{\mathrm{c}}$ describes the relative velocity at the free surface and is given by (Rusche, 2002):

$$
\boldsymbol{V}_{c}=\mathbf{n}_{f} \min \left[C_{\alpha} \frac{\phi_{f}}{S_{f}}, \max \left(\frac{\phi_{f}}{S_{f}}\right)\right]
$$

where the coefficient $C_{\alpha}$ set to 1 controls the level of compression at the interface, and $\phi_{f}$ and $S_{f}$ correspond to the mass flux and cell surface area, respectively. The use of the compression scheme avoids the geometrical reconstruction traditionally performed on VOF method. Furthermore the algebraic method adopted here is readily extensible to unstructured meshes.

The physical properties in any numerical cell of the domain can be expressed through the liquid fraction as follows:

$$
\chi=\alpha \chi_{\text {liquid }}+(1-\alpha) \chi_{\text {gas }}
$$

where $\chi$ stands for any physical properties involved in the problem, e.g., the density and viscosities for both the solvent and the polymer.

The interaction with the substrate is handled through the dynamics contact angle. The accuracy in droplet dynamics is highly related to the way the dynamics contact angle is modeled. Here the dynamics contact angle $\left(\theta_{d}\right)$ is implemented using the correlation by Kistler, (1993):

$$
\theta_{d}=f_{H}\left[C a+f_{H}^{-1}\left(\theta_{E}\right)\right]
$$


the Hoffman function is given by :

$$
f_{H}(\mathrm{~s})=\arccos \left\{1-2 \tanh \left[5.16\left[\frac{s}{1+1.31 s^{0.99}}\right]^{0.706}\right]\right\}
$$

$U_{c l}$ is the contact line velocity which is approximated by using the velocity at the interface in the first computational point above the wall. In order to account for the hysteresis effect in the numerical model, the equilibrium contact angle $\theta_{E}$ in (9) is replaced by either the advancing contact angle, $\theta_{A}$, or the receding contact angle $\theta_{R}$ depending on the direction of the velocity at the contact line. This implementation makes the model very sensitive to the surface hysteresis as well, which plays a major role in the modeling of droplet impact behavior (Yokoi et al. 2009). Unlike in (Yokoi et al. 2009) the present Kistler modified model does not rely heavily on the experimental measurement of the dynamics contact angle evolution for each simulating case of droplet impact. We discussed the accuracy of this approach in our previous publication (Moghtadernejad and Tembely et al. 2015). It worth noting that most of the simulation regarding non-Newtonian fluid overlook the crucial role of the dynamics contact on droplet impact.

\section{Fluids and Substrates Properties}

In order to test the model the viscoelastic fluid and its properties are based on the experimental set up of the filament stretching and thinning, extensional rheometer, the so-called "Cambridge Trimaster". This apparatus, which has been presented in Vadillo et al. (2010), performs filament stretching at a constant velocity for a fluid initially placed between two pistons of initial diameter $D_{0}$ of $1.2 \mathrm{~mm}$.

Both pistons, are attached on the opposite side of a belt, and move symmetrically apart for a given distance allowing the mid-filament to remain in a central position. The subsequent measurement of 
the filament mid-diameter enables to accurately determine the relaxation time of the fluid, which the authors previously showed is essential in predicting numerically the filament thinning behavior.

The simulation considers the case of the Newtonian solvent which is diethyl phthalate (DEP), in addition to the case of polymer solutions Polystyrene (PS), $110000 \mathrm{~g} / \mathrm{mol}$, dissolved into the DEP solvent.

The physical properties of the fluids are presented in Table 1.

\section{Table 1}

Fluids physical properties

\begin{tabular}{cccc}
\hline \hline Fluids & Surface & Viscosity & Density \\
& Tension & $(\mathrm{mPa} . \mathrm{s})$ & $\left(\mathrm{kg} / \mathrm{m}^{3}\right)$ \\
& $(\mathrm{mN} / \mathrm{m})$ & & \\
\hline DEP & 37 & 14 & 1120 \\
\hline DEP+2.5\%PS & 37 & 31 & 1120 \\
\hline \hline
\end{tabular}

In addition the relaxation time deduced for the polymer solution, $\mathrm{DEP}+2.5 \mathrm{wt} \% \mathrm{PS}$, is $\lambda=1.19 \mathrm{~ms}$, while the extensibility, based on the fluids physical properties, is found to be $L=15$ (Tembely et al. 2012). Furthermore, two types of substrates are considered for the numerical simulation: a hydrophilic and superhydrophobic, which properties are very similar to the aluminum and WX2100 properties for water droplet, respectively (Graham et al., 2012). Experimentally, it may be challenging to find a 
substrate exhibiting those chemical properties with respect the DEP and DEP $+2.5 \mathrm{wt} \% \mathrm{PS}$, though the aim of this study is to highlight the features of viscoelasticity in droplet dynamics, which is numerically-poorly documented in the literature, especially with the added effect of substrate wetting. The contact angles (CA) consisting of the equilibrium contact angles, in addition to the hysteresis with the advancing and receding contact angles of these substrates, are given in Table 2.

Table 2

Substrates contact angle (CA)

\begin{tabular}{lccc}
\hline \hline Substrate & Equilibrium & Advancing & Receding \\
& $\mathrm{CA}\left({ }^{\circ}\right)$ & $\mathrm{CA}\left({ }^{\circ}\right)$ & $\mathrm{CA}\left({ }^{\circ}\right)$ \\
& & & \\
& & & \\
Hydrophilic & 74 & 90 & 50 \\
\hline & & & \\
Superhydrophobic & 154 & 162 & 142 \\
\hline \hline
\end{tabular}

\section{Numerical Results}

The governing equations are implemented in OpenFOAM/C++ opensource code. The PIMPLE algorithm combines the Pressure Implicit with Splitting of Operators (PISO) and the SIMPLE algorithms is used to calculate the pressure and velocity fields while the viscoelastic constitutive equation are solved using a preconditioned bi-conjugate gradient technique. We consider a droplet, Newtonian or viscoelastic modeled by FENE-CR, of diameter $D_{0}=1 \mathrm{~mm}$, impacting at $V_{0}=1 \mathrm{~m} / \mathrm{s}$ on a horizontal substrate of different wettability. The computational domain consisting of 50000 cells, with refinement near the droplet of about 50 cells per diameter (Fig.1). The simulations are performed 
in parallel using domain decomposing method, each processor handling one subdomain.

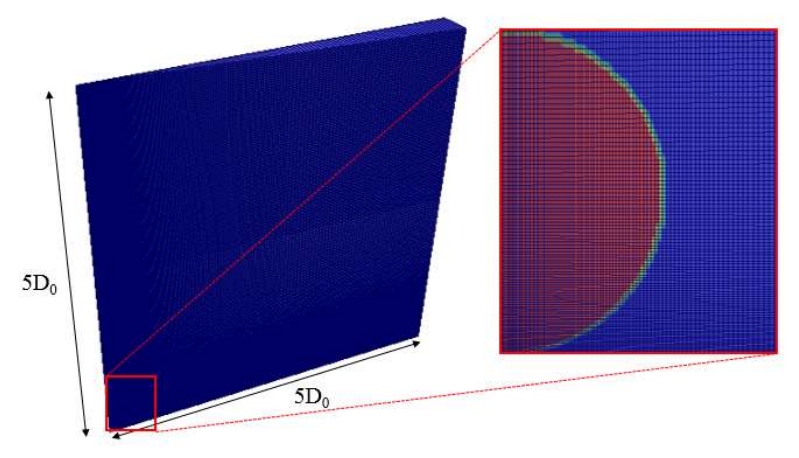

Fig. 1. Computational domain mesh for the viscoelastic droplet impact.

\subsection{Impact on hydrophilic substrate}

We numerically investigate both the Newtonian and non-Newtonian droplet impact on a hydrophilic substrate. The simulated sequence images of the impact of a $1 \mathrm{~mm}$ diameter droplet at $1 \mathrm{~m} / \mathrm{s}$ on a hydrophilic substrate is depicted in Fig.2. The right-hand side corresponds to the Newtonian fluid while the left hand-side represents the viscoelastic fluid modeled by the FENE-CR viscoelastic model. The kinematic phase (up to $t V_{0} / D_{0}=1$ ) seems to be independent of the fluid model, as highlighted in Fig.3, regarding the evolution of the spreading diameter. Though, the subsequent phase of the droplet spreading indicates a much more pronounced difference between the Newtonian and viscoelastic model. In addition, the viscoelastic fluid shows little oscillation for the droplet height, while having a greater maximum spreading diameter, due to the dominance of the elastic normal stress over of the surface tension, which favors retraction. Furthermore, the liquid may flow out of the droplet center towards its periphery, much like as in the filament stretching and thinning problem (Tembely et al., 2012). 


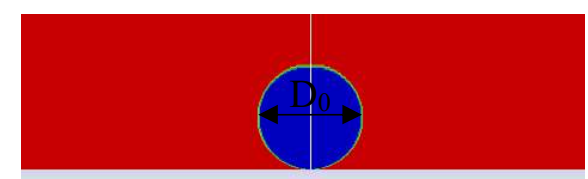

Time: 0.000000 s

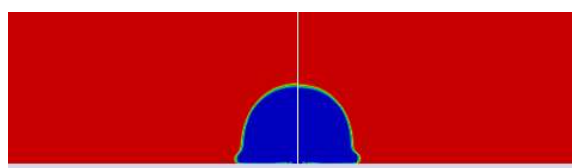

Time: $0.000250 \mathrm{~s}$

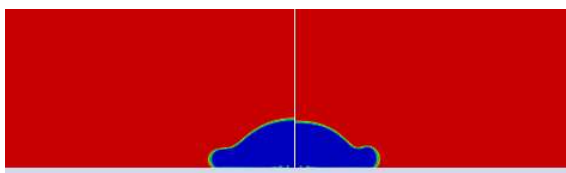

Time: $0.000600 \mathrm{~s}$

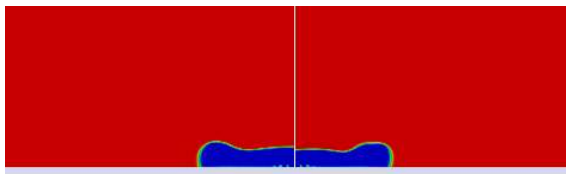

Time: $0.001200 \mathrm{~s}$

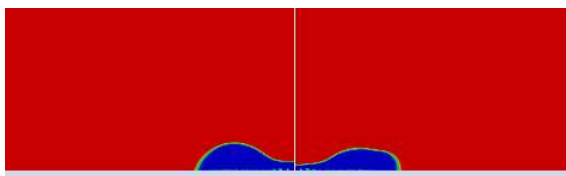

Time: $0.002200 \mathrm{~s}$

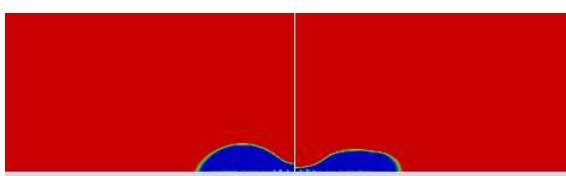

Time: $0.002450 \mathrm{~s}$

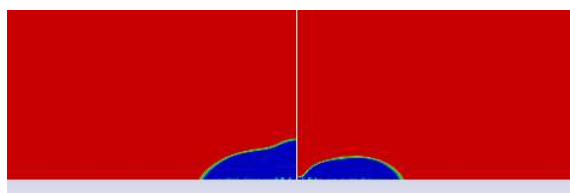

Time: 0.003000 s 


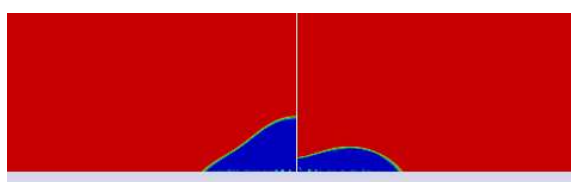

Time: 0.004000 s

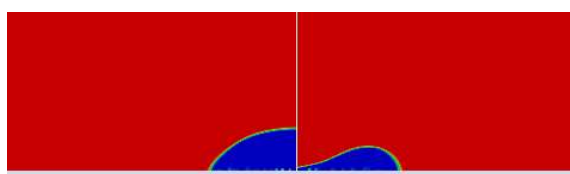

Time: 0.006000 s

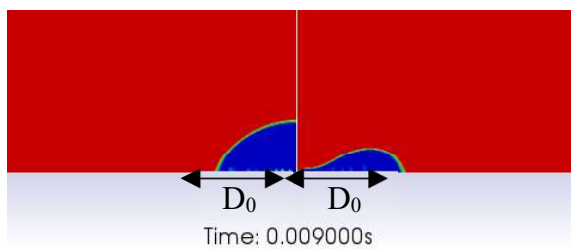

Fig. 2. Fig. 4. Simulated comparison between (right) the Newtonian solvent DEP and (left) viscoelastic polymer solution, $\mathrm{DEP}+2.5 \mathrm{wt} \% \mathrm{PS}$, on the impact of a $1 \mathrm{~mm}$ diameter droplet at $1 \mathrm{~m} / \mathrm{s}$ on the hydrophilic substrate.

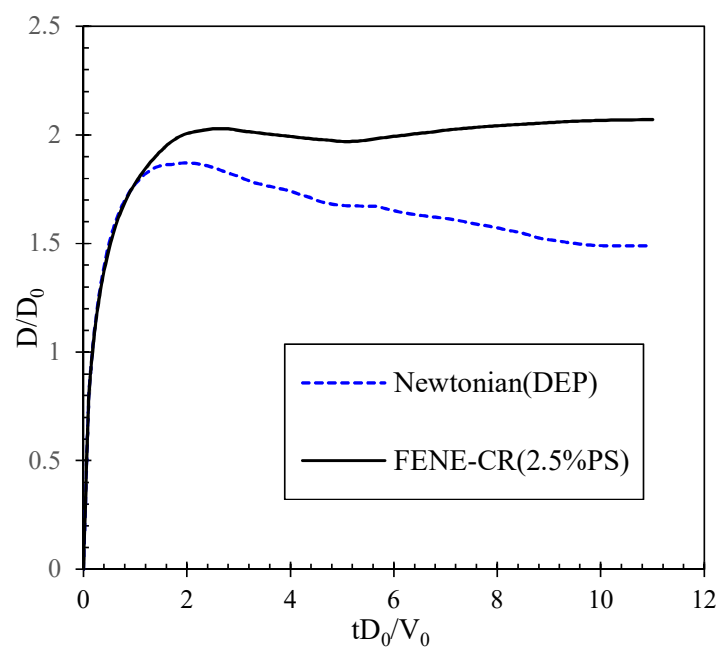

Fig. 3. Simulated spreading diameter evolution of the droplet Impact on the Hydrophilic substrate. 


\subsection{Impact on a superhydrophobic substrate}

The comparison between droplet impact of a Newtonian and viscoelastic fluid by FENE-CR is shown in Fig .4, the image sequences correspond to the impact on superhydrophobic substrate of a $1 \mathrm{~mm}$ droplet impacting at $1 \mathrm{~m} / \mathrm{s}$. The left-hand side corresponds to the Newtonian solvent while the righthand side represents the polymer solution modeled by the FENE-CR viscoelastic model. The addition of a minute quantity of polymer to a Newtonian liquid is found to affect droplet dynamics mainly during the recoiling stage for the impact conditions that have been investigated in the present work. The kinetic energy of the impacting droplet is converted to the elastic and surface energy; that stored energy contributes to droplet retraction and rebound on superhydrophobic surface, after some dissipation by the viscosity. As foreseeable, the Newtonian (DEP) droplet rebounds and detaches after impact on the superhydrophobic substrate, however, the rebound is suppressed with the dilute polymer solution $(\mathrm{DEP}+2.5 \mathrm{wt} \% \mathrm{PS})$, where the high elongational viscosity dissipates much of the drop kinetic energy during the spreading phase. The contrast between the Newtonian and the polymer solution behavior is quantified in Fig. 5 with the spreading diameter evolution. For superhydrophobic substrate, it is worth mentioning not to confuse the maximum spreading diameter in contact with substrate to the maximum (deformation) diameter. The latter is higher for viscoelastic fluid than the Newtonian one (Fig .4). In addition, we found that with a much lower concentration of polymer the droplet bounces back again on the superhydrophobic substrate, which exhibits per se a higher retraction energy (Fig.5). The presented model using a single FENE-CR model should be extended to multimode, or other constitutive equations, along with tailored experiments for a full description of viscoelastic droplet impact. 


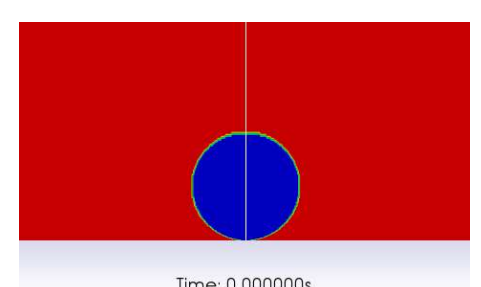

Time: $0.000000 \mathrm{~s}$

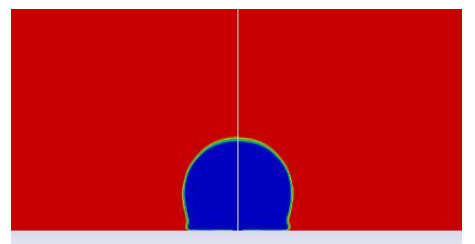

Time: $0.000150 \mathrm{~s}$

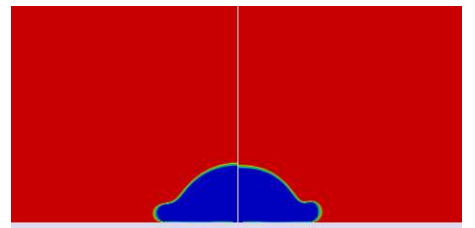

Time: $0.000500 \mathrm{~s}$

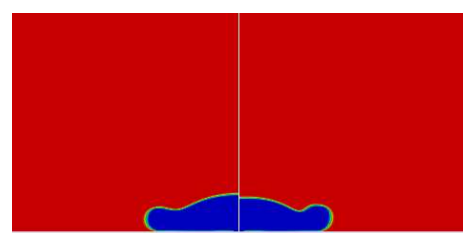

Time: 0.000800 s

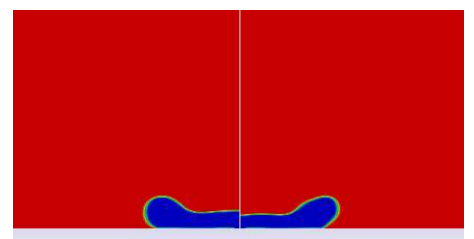

Time: $0.001400 \mathrm{~s}$

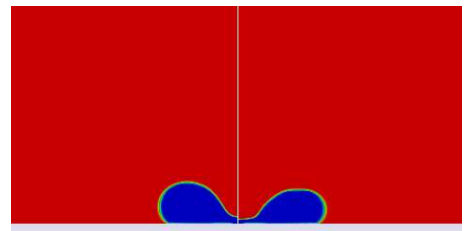

Time: $0.002250 \mathrm{~s}$

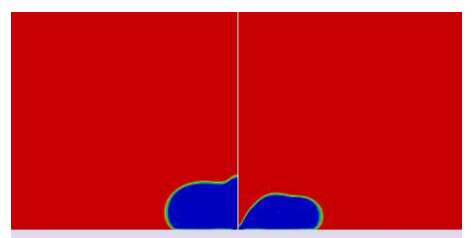

Time: $0.002500 \mathrm{~s}$ 


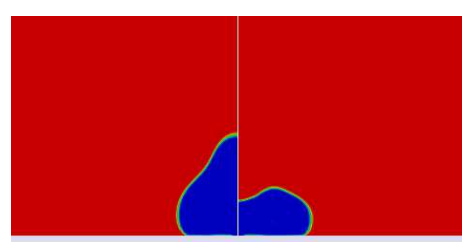

Time: $0.003000 \mathrm{~s}$

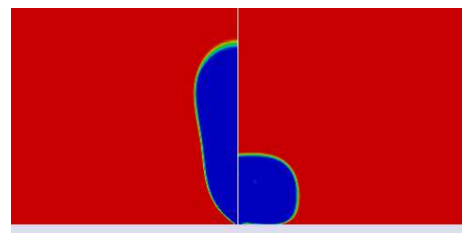

Time: 0.005300 s

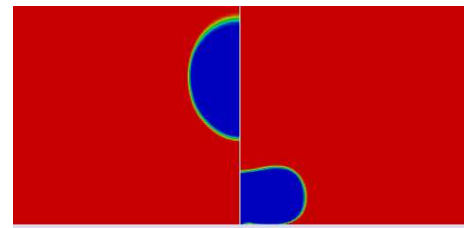

Time: $0.007000 \mathrm{~s}$

Fig. 4. Simulated comparison between (right) the Newtonian solvent DEP and (left) viscoelastic polymer solution, $\mathrm{DEP}+2.5 \mathrm{wt} \% \mathrm{PS}$, on the impact of a $1 \mathrm{~mm}$ diameter droplet at $1 \mathrm{~m} / \mathrm{s}$ on the superhydrophobic substrate.

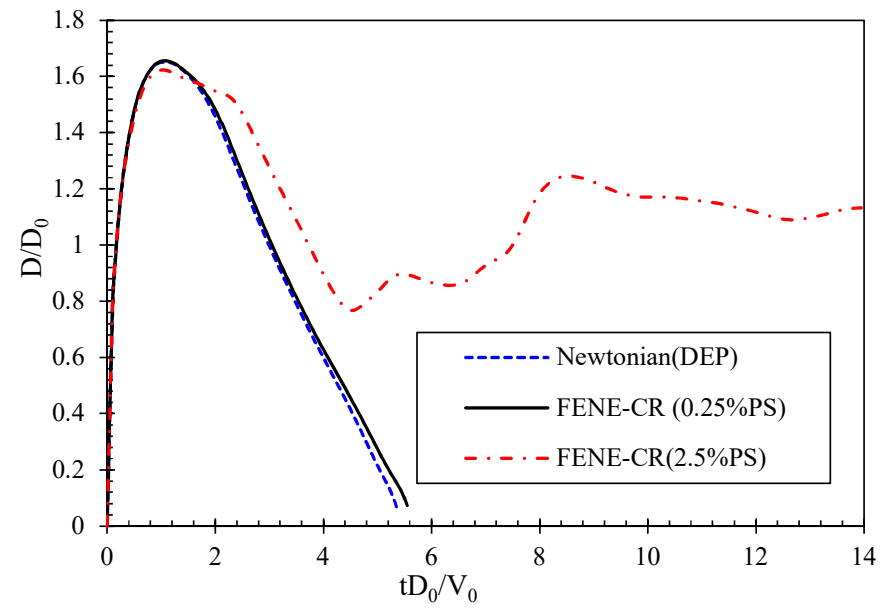


Fig.5. Simulated spreading diameter of a $1 \mathrm{~mm}$ droplet impact at $1 \mathrm{~m} / \mathrm{s}$ of a Newtonian (DEP) fluid and two polymer solutions, $\mathrm{DEP}+0.25 \%$, DEP $+2.5 \% \mathrm{PS}$.

\section{Conclusion}

A volume of fluid (VOF) method is developed to perform a comparative study of Newtonian and viscoelastic droplet impact on different substrates. The interaction with the substrate is handled with a more realistic dynamics contact angle, as opposed to a static contact angle. The constitutive equations considered for the viscoelastic fluids is the FENE-CR model. While any significant difference is found on droplet impact up until the kinematic phase, the substrate and the fluid viscoelasticity influence become much more dominant on droplet dynamics during the recoiling phase. The simulation indicates that the dilute polymer solution droplet has higher spreading diameter compared to Newtonian solvent on a hydrophilic substrate, which results from both the kinetic energy dissipation and the fact that the elastic normal stress overcomes the surface tension force which favors droplet retraction. In addition, it is inferred the existence of polymer concentration at which the droplet may longer detach even on superhydrophobic substrate. The capability of the model to retrieve such a feature is of particular interest for processes ranging from inkjet, spray cooling, to pesticide or nutrient deposited as spray on plants.

\section{References}

Amarouchene Y., Bonn D., Meunier J and Kellay H, 2001. Inhibition of the Finite-Time Singularity during Droplet Fission of a Polymeric Fluid. Phys. Rev. Lett. 863558. 
Chilcott M. D., and Rallison J. M.,1988. Creeping flow of dilute polymer solutions past cylinders and spheres. J. Non-Newtonian Fluid Mech. 29, 381-432.

Clemens W., Fix W., Ficker J., Knobloch A., and Ullmann A.2004. From polymer transistors toward printed electronics. J. Mater. Res., 19,1963-1973.

Crochet M.J., Keunings R.1982. Finite element analysis of die swell of a highly elastic fluid, J. NonNewtonian Fluid Mech. 10, 339-356.

Dong, H., Carr W. W., and Morris, J. F., 2006. An experimental study of drop-on-demand drop formation. Phys. Fluids 18, 072102.

Fang J., Owens, R.G., Tacher L., Parriaux, A., 2006. A numerical study of the SPH method for simulating transient viscoelastic free surface flows, J. Non-Newt. Fluid Mech. 139, 68-84.

Graham P.,Farhangi M.M., A Dolatabadi A., 2012. Dynamics of droplet coalescence in response to increasing hydrophobicity. Physics of Fluids, 24 (11), 112105.

Hoath, S.D., Vadillo, D.C., Oliver G. Harlen, O.G, McIlroy C., Neil F. Morrison, O.F., Hsiao W.K., Tuladhar T.R., Jung S., Martin G.D.,. Hutchings I.M., 2014. Inkjet printing of weakly elastic polymer solutions. Journal of Non-Newtonian Fluid Mechanics, 205, 1-10.

Kistler S. F.. In Hydrodynamics of wetting”, Berg, J. C., Ed.;Wettability; Marcel Dekker Inc.: New York; pp 311-429, 1993. 
S. Moghtadernejad, M. Tembely, M. Jadidi, and A. Dolatabadi "Shear driven droplet shedding and coalescence on a superhydrophobic surface” Physics of Fluids 27 (3), 032106, (2015)

Morrison, N. F., and Harlen O. G. 2010. Viscoelasticity in inkjet printing. Rheol. Acta 49, 619-632.

M. Mohammadi, M. Tembely, and A. Dolatabadi, "Predictive Model of Supercooled Water Droplet Pinning/Repulsion Impacting a Superhydrophobic Surface: The Role of the Gas-Liquid Interface" Temperature, Langmuir, 2017, 33 (8), pp 1816-1825, DOI: 10.1021/acs.langmuir.6b04394

Rusche H.. Computational Fluid Dynamics of Dispersed Two Phase Flows at High Phase Fractions. PhD Thesis, Imperial College University of London, 2002.

Singh M., Haverinen H.M, Dhagat P., Jabbour G. E. 2010. Inkjet Printing Process and Its Applications. Adv. Mater. 22, 673-685.

Tome M.F., Mangiavacchi N., Cuminato J.A., Castelo A., McKee, S. 2002. A finite difference technique for simulating unsteady viscoelastic free surface flows, J. Non-Newtonian Fluid Mech. 106 (2002) 61-106.

Tomé M.F., Paulo G.S., Pinho F.T., Alves M.A. 2010. Numerical solution of the PTT constitutive equation for unsteady three-dimensional free surface flows, J. Non-Newt. Fluid Mech. 165, 247-262.

Tembely D., Vadillo D. C., Mackley M. R., Soucemarianadin A., 2012. The matching of a "onedimensional" numerical simulation and experiment results for low viscosity Newtonian and nonNewtonian fluids during fast filament stretching and subsequent break-up. J. Rheol. 56(1), 159-183, 
2012.

Vadillo, D. C., Tuladhar T. R., Mulji A. C., Jung, S., Hoath S. D., and Mackley, M.R., 2010. “Evaluation of the inkjet fluid's performance using the 'Cambridge Trimaster' filament stretch and break-up device,” J. Rheol. 54(2), 261-282.

Xu, J., Attinger, D., 2008. Drop on demand in a microfluidic chip. J. Micromech. Microeng. 18 065020.

Yokoi K., Vadillo D., John Hinch J., Ian Hutchings, I., 2009. Numerical studies of the influence of the dynamic contact angle on a droplet impacting on a dry surface, Phys. Fluids, 21, 072102.

Yu, J.-D., Sakai, S., Sethian, J.A. 2007. Two-phase viscoelastic jetting. Journal of Computational Physics 220, 568-585

Yue P., J.J. Feng J.J. 2012. Phase-field simulations of dynamic wetting of viscoelastic fluids. Journal of Non-Newtonian Fluid Mechanics 189-190, 8-13. 